\title{
The Role of Serum Cholesterol Level in the Pathogenesis of Major Depressive Disorder
}

\section{Madhura TK*}

Department of Biochemistry, Defense Institute of Advanced Technology, Near Khadakwasla Dam, Girinagar, Pune, India

\section{Commentary}

\section{Aims and Objectives}

1. To determine the level of serum cholesterol in patients with major depressive disorder

2. To correlate the serum cholesterol level to the role of pathogenesis in major depressive disorder.

\section{Introduction}

Major Depressive Disorder is a condition characterized by one or more Major Depressive Episodes without a history of Manic, Mixed, or Hypomanic Episodes. These Major Depressive Episodes are not due to a medical condition, medication, abused substance, or Psychosis. If Manic, Mixed, or Hypomanic Episodes develop, the diagnosis is changed to Bipolar Disorder. The Major Depressive Episode must have either depressed mood or loss of interest.

\section{Diagnostic Considerations}

The differential diagnosis for depression includes a wide variety of medical disorders, such as the following:

- Central nervous system diseases (eg, Parkinson disease, dementia, multiple sclerosis, neoplastic lesions)

- Endocrine disorders (eg, hyperthyroidism, hypothyroidism)

- Drug-related conditions (eg, cocaine abuse, side effects of some CNS depressants)

- Infectious disease (eg, mononucleosis)

- $\quad$ Sleep-related disorders

\section{Related Psychiatric Disorders}

Major depressive disorder must be differentiated from dysthymia. Patients with dysthymia present with low mood for at least 2 years as a primary symptom; they have insufficient symptoms to meet criteria for major depressive disorder. However, dysthymia may predate a depressive episode.

Misdiagnosis of bipolar disorder as recurrent unipolar depression may occur if the clinician does not identify the presence of hypomania between depressive episodes. This leads to inadequate treatment and, theoretically, could lead to a precipitation of a hypomanic, manic, or mixed episode.

Patients with anxiety disorders are at higher risk for developing comorbid depression. In such patients, it is important to identify the anxiety disorder, because affected individuals often require specific treatment approaches. Commonly encountered anxiety disorders include the following:

\section{Generalized Anxiety Disorder}

Obsessive-compulsive disorder

Panic disorder

Phobic disorders

\section{Posttraumatic stress disorder}

Patients with certain personality disorders (eg, borderline personality disorder) may present with mood changes as a prominent symptom. Remember that the presence of a personality disorder can be difficult to determine in the setting of active affective symptoms. Many depressed patients who appear labile, demanding, or pathologically dependent look dramatically different once the depressive episode has been treated adequately.

People with eating disorders also have a high rate of comorbid major depressive disorder and require specific treatment approaches. These disorders include bulimia, anorexia nervosa, and eating disorder not otherwise specified. A large percentage of individuals in this last group have binge-eating disorder.

\section{Central Nervous System Disorders}

Major depressive disorder does not cause focal neurologic signs. Such findings should prompt an evaluation for other organic syndromes.

A broad range of physiologic and structural CNS processes can produce changes in mood and behavior. Note that major depressive disorder can produce measurable cognitive deficits or a worsening of preexisting dementia. This decline in cognitive functioning, which on formal testing appears to arise from impaired concentration or motivation, is referred to as pseudo dementia or as dementia of depression and should remit with successful treatment of the depressive episode.

Alzheimer disease and other degenerative and vascular dementias can be associated with affective symptoms, especially in the initial phases of dementia. Mood disorders are also very prominent in Parkinson disease, Huntington disease, multiple sclerosis, stroke, and seizure disorders. Neoplastic lesions of the CNS can cause changes in mood and behavior before the onset of focal neurologic signs.

\section{Endocrine Disorders}

Endocrinologic disorders involving the hypothalamicpituitary-adrenal axis or thyroid are especially likely to produce changes in mood. These include Addison disease, Cushing syndrome, hyperthyroidism, hypothyroidism, prolactinomas, and hyperparathyroidism.

*Corresponding author: Madhura TK, Defense Institute of Advanced Technology, Near Khadakwasla dam, Girinagar, Pune 411025, India, Tel: 9921041635; E-mail: madhuratksharma@gmail.com

Received: November 30, 2016; Accepted: January 25, 2016; Published January 28,2016

Citation: Madhura TK (2016) The Role of Serum Cholesterol Level in the Pathogenesis of Major Depressive Disorder. Biochem Anal Biochem 5: 241 doi:10.4172/2161 1009.1000241

Copyright: (c) 2016 Madhura TK. This is an open-access article distributed under the terms of the Creative Commons Attribution License, which permits unrestricted use, distribution, and reproduction in any medium, provided the original author and source are credited. 


\section{DSM-IV Criteria for Major Depressive Disorder (MDD)}

I. Depressed mood or a loss of interest or pleasure in daily activities for more than two weeks.

II. Mood represents a change from the person's baseline.

III. Impaired function: social, occupational, educational.

IV. Specific symptoms, at least 5 of these 9 , present nearly every day:

1. Depressed mood or irritable most of the day, nearly every day, as indicated by either subjective report (e.g., feels sad or empty) or observation made by others (e.g., appears tearful).

2. Decreased interest or pleasure in most activities, most of each day

3. Significant weight change (5\%) or change in appetite

4. Change in sleep: Insomnia or hypersomnia

5. Change in activity: Psychomotor agitation or retardation

6. Fatigue or loss of energy

7. Guilt/worthlessness: Feelings of worthlessness or excessive or inappropriate guilt

8. Concentration: diminished ability to think or concentrate, or more indecisiveness

9. Suicidality: Thoughts of death or suicide, or has suicide plan

\section{Concept behind the Study}

Cholesterol is the vital component of eukaryotic cell membranes and plays a crucial role maintaining cellular function, membrane dynamics, transport across cell membranes and physical protection. The alteration in membrane physical properties is due to interaction of cholesterol moiety with varies protein components in cellular membranes. The heterogeneity in cholesterol distribution in membrane domains sequester proteins contributing to significance membrane function. The seven transmembrane domain G-protein coupled receptors (GPCRs) are among the largest protein families in mammals and represent $2 \%$ of the total proteins coded by the human genome. They mediate transduction events, which are the primary means of cellular communication and response to external environment. The recently reported role of cholesterol in the function and organization of the neuronal serotonin1A receptor, a representative of the GPCR family which is present endogenously in the hippocampal region of the brain, is highlighted. The neurotransmitter release at the synapses underlies fundamental brain activities such as cognition, emotion, and memory. It is therefore natural to speculate that the neurotransmitter release depends on the cholesterol level in the brain. A low cholesterol level may alter the pattern of the release, which in turn perturbs the brain activities to the extent that it could lead to behavioral dysfunction, depression, suicide, and memory loss.

There exists an elaborate assembly of protein and lipid components, releasing the transmitter from pre-synaptic neurons. Cholesterol is the vital component of exocytosis apparatus playing a crucial role in biogenesis and transport of neurotransmitters and other substances in synaptic vesicles. Cholesterol can affect synaptic membrane fusion in a number of different ways:

(i) Cholesterol is proposed to form laterally separated membrane domains called rafts', in cooperation with another brain lipid sphingolipid. The rafts could play a role in driving 58 the clustering of SNAREs into a specific membrane site, which may be necessary for productive fusion. (ii) it is shown that cholesterol induces the clustering of the SNARE proteins by promoting self-assembly of the proteins without the rafts. The clustering of SNAREs is thought to be helpful for docking and fusion of vesicles.

(iii)due to its unique inverse wedge like molecular shape, cholesterol is thought to play a role in membrane fusion which involved curving and bending of the bilayers.

Since serum cholesterol level is in equivalence with neuronal cholesterol, the reduced cholesterol levels in serum disrupts the neuronal levels leading to impaired neurotransmitter release (eg. Serotonin), thus precipitating the symptoms of depression.

\section{Case Selection}

\section{Inclusion criteria}

Clinical Diagnosis made based upon DSM IV criteria

Age : between $15-45$ years age group

Sex : both males and female cases

\section{Exclusion criteria}

Endocrine disorders: metabolic syndrome, Obesity

If patient is known case of Polycystic ovarian syndrome, Diabetes mellitus Type 1 and 2, Hypothyroidism and hyperthyroidism, cushing' $\mathrm{s}$ syndrome, dyslipidemia.

Central nervous system diseases (eg, Parkinson disease, dementia, multiple sclerosis, neoplastic lesions)

\section{Substance abuse}

smoking and alcoholism or any other kind of addiction.

- On therapeutic drugs: Antihypertensive medications (especially reserpine and methyldopa)

- Smoking-cessation aids (eg, varenicline)

- Steroids

- Sex hormones and medications that affect sex hormones (eg, estrogen, progesterone, testosterone, gonadotropin-releasing hormone $[\mathrm{GnRH}]$ antagonists)

- $\mathrm{H} 2$ blockers (eg, ranitidine, cimetidine)

- Sedatives

- Muscle relaxants

- Appetite suppressants

- Chemotherapy agents (eg, vincristine, procarbazine, L-asparaginase, interferon, vinblastine)

- Central nervous system diseases (eg, Parkinson disease, dementia, multiple sclerosis, neoplastic lesions)

\section{Infrastructure needed}

The parameter under the present study can be done using fully auto analyser / semi-auto analyser at biochemistry lab Yellur Hospital.

\section{Budget}

This study needs sample collection at KLE CC hospital \& analyzing at Lab Yellur Hospital.

Kit for analysis of total cholesterol level is required. 